\title{
A refinement of an inequality due to Ankeny and Rivlin
}

\author{
Dinesh Tripathi
}

Abstract. Let $p(z)=\sum_{\nu=0}^{n} a_{\nu} z^{\nu}$ be a polynomial of degree $n$,
\[ M(p, R):=\max _{|z|=R \geq 0}|p(z)| \text {, and } M(p, 1):=M(p) . \]

Then by well-known result due to Ankeny and Rivlin [1], we have

$$
M(p . R) \leq\left(\frac{R^{n}+1}{2}\right) M(p), R \geq 1 .
$$

In this paper, we sharpen and generalizes the above inequality by using a result due to Govil [5].

Mathematics Subject Classification (2010): 15A18, 30C10, 30C15, 30A10.

Keywords: Inequalities, polynomials, maximum modulus.

\section{Introduction}

Let $\mathcal{P}_{n}:=\left\{p(z) ; p(z)=\sum_{\nu=0}^{n} a_{\nu} z^{\nu}\right\}$ be a class of polynomial of degree $n$. Let $\max _{|z|=R}|p(z)|=M(p, R)$ and $M(p, 1)=M(p)$. Then from maximum modulus principle, $M(p, R)$ is a strictly increasing function and for $0 \leq R<\infty$. Also, it is a simple deduction from the maximum modulus principle (see [10, p. 158, Problem 269]) that for $R \geq 1$,

$$
M(p, R) \leq R^{n} M(p) .
$$

The result is best possible and equality holds if and only if $p(z)=\lambda z^{n}$, where $\lambda$ being a complex number.

For $p \in \mathcal{P}_{n}$ not vanishing in the interior of unit circle, Ankeny and Rivlin [1] sharpened inequality (1.1), by proving following result. 
Theorem 1.1. If $p \in \mathcal{P}_{n}$ and $p(z) \neq 0$ for $|z|<1$, then for $R \geq 1$,

$$
M(p, R) \leq\left(\frac{R^{n}+1}{2}\right) M(p), R \geq 1 .
$$

The above inequality is sharp and equality holds for polynomial

$$
p(z)=\alpha+\beta z^{n},|\alpha|=|\beta| .
$$

Since the equality in (1.2) holds only for $p(z)=\alpha+\beta z^{n}$, which satisfy

$$
|\beta|=\frac{1}{2} M(p),
$$

therefore it should possible to improve the bound (1.2) for the polynomial not satisfying (1.3). Govil [5] solve this problem by proving the following result.

Theorem 1.2. If $p \in \mathcal{P}_{n}$ and $p(z) \neq 0$ for $|z|<1$, then for $R \geq 1$,

$$
\begin{aligned}
M(p, R) \leq & \left(\frac{R^{n}+1}{2}\right) M(p)-\frac{n}{2}\left(\frac{M(p)^{2}-4\left|a_{n}\right|^{2}}{M(p)}\right)\left\{\frac{(R-1) M(p)}{M(p)+2\left|a_{n}\right|}\right. \\
& \left.-\ln \left(1+\frac{(R-1) M(p)}{M(p)+2\left|a_{n}\right|}\right)\right\} .
\end{aligned}
$$

The result is best possible and the equality holds for $p(z)=\left(\lambda+\mu z^{n}\right), \lambda$ and $\mu$ being complex numbers with $|\lambda|=|\mu|$.

The other extension and generalization of Theorem 1.1 has been mentioned in the various article,e.g Aziz [2], Aziz and Mohammad [3], Milovanović, Mitrinović and Rassias [8], Govil [6], Govil, Qazi and Rahman [7] and Rahman and Schmeisser [12], Tripathi [13] etc.

\section{Main results}

In this paper, we prove the following improved generalization of Theorem 1.2 for the class of Lacunary type of polynomial

$$
p(z)=a_{0}+\sum_{\nu=\mu}^{n} a_{\nu} z^{\nu}
$$

Theorem 2.1. If $p(z)=a_{0}+\sum_{\nu=\mu}^{n} a_{\nu} z^{\nu}$ is a polynomial of degree $n$ and $p(z) \neq 0$ for $|a|<k, k \geq 1$, then for $R>r \geq 1$,

$$
\begin{aligned}
\left|\left\{p\left(R e^{i \theta}\right)\right\}^{s}\right| & \leq \frac{\left(R^{n s}-r^{n s}\right)}{1+k^{\mu}}\{M(p)\}^{s}-\frac{n}{1+k^{\mu}}\{M(p)\}^{s}\left(1-\frac{\left(1+k^{\mu}\right)\left|a_{n}\right|}{M(p)}\right) h(n) \\
& +\left|\left\{p\left(r e^{i \theta}\right)\right\}^{s}\right|
\end{aligned}
$$


where

$$
\begin{aligned}
h(n) & =\left(\frac{R^{n}-r^{n}}{n}\right)+\sum_{k=1}^{n-1}\left(\frac{R^{n-k}-r^{n-k}}{n-k}\right)(-1)^{k}\left(\frac{\left(1+k^{\mu}\right)\left|a_{n}\right|}{M(p)}+1\right)\left(\frac{\left(1+k^{\mu}\right)\left|a_{n}\right|}{M(p)}\right)^{k-1} \\
& +(-1)^{n}\left(\frac{\left(1+k^{\mu}\right)\left|a_{n}\right|}{M(p)}+1\right)\left(\frac{\left(1+k^{\mu}\right)\left|a_{n}\right|}{M(p)}\right)^{n-1} \ln \left(\frac{R(M(p))+\left(1+k^{\mu}\right)\left|a_{n}\right|}{r(M(p))+\left(1+k^{\mu}\right)\left|a_{n}\right|}\right)
\end{aligned}
$$

for $n \geq 1$ and $h(0)=0$.

On taking $s=0, \mu=1, r=1$ and $k=1$, we have the following application of above Theorem 2.1.

Corollary 2.2. If $p \in \mathcal{P}_{n}$ and $p(z) \neq 0$ for $|z|<1$, then for $R \geq 1$,

$$
\left|p\left(R e^{i \theta}\right)\right| \leq \frac{\left(R^{n}+1\right)}{2} M(p)-\frac{n}{2} M(p)\left(1-\frac{2\left|a_{n}\right|}{M(p)}\right) h(n),
$$

where

$$
\begin{aligned}
h(n) & =\left(\frac{R^{n}-1}{n}\right)+\sum_{k=1}^{n-1}\left(\frac{R^{n-k}-1}{n-k}\right)(-1)^{k}\left(\frac{2\left|a_{n}\right|}{M(p)}+1\right)\left(\frac{2\left|a_{n}\right|}{M(p)}\right)^{k-1} \\
& +(-1)^{n}\left(\frac{2\left|a_{n}\right|}{M(p)}+1\right)\left(\frac{2\left|a_{n}\right|}{M(p)}\right)^{n-1} \ln \left(1+\frac{(R-1) M(p)}{M(p)+2\left|a_{n}\right|}\right)
\end{aligned}
$$

for $n \geq 1$ and $h(0)=0$.

Remark 2.3. From Lemma 3.7, we get $0 \leq h(n)$. Using this in Corollary 2.2, we get

$$
\left|p\left(R e^{i \theta}\right)\right| \leq \frac{\left(R^{n}+1\right)}{2} M(p)-\frac{n}{2} M(p)\left(1-\frac{2\left|a_{n}\right|}{M(p)}\right) h(n) \leq \frac{\left(R^{n}+1\right)}{2} M(p),
$$

which shows that Corollary 2.2, clearly refines Theorem 1.1 due to Ankeny and Rivlin [1].

Remark 2.4. From Lemma 3.7, we have $h(1) \leq h(n)$. Using this inequality in Corollary 2.2 , we get

$$
\begin{aligned}
\left|p\left(R e^{i \theta}\right)\right| & \leq \frac{\left(R^{n}+1\right)}{2} M(p)-\frac{n}{2} M(p)\left(1-\frac{2\left|a_{n}\right|}{M(p)}\right) h(n) \\
& \leq \frac{\left(R^{n}+1\right)}{2} M(p)-\frac{n}{2} M(p)\left(1-\frac{2\left|a_{n}\right|}{M(p)}\right) h(1),
\end{aligned}
$$

and,

$$
h(1)=(R-1)-\left(1+\frac{2\left|a_{n}\right|}{M(p)}\right) \ln \left(1+\frac{(R-1) M(p)}{M(p)+2\left|a_{n}\right|}\right) .
$$

Substitute the value of $h(1)$ in $(2.3)$, we get

$$
\begin{aligned}
\left|p\left(R e^{i \theta}\right)\right| \leq & \left(\frac{R^{n}+1}{2}\right) M(p)-\frac{n}{2}\left(\frac{M(p)^{2}-4\left|a_{n}\right|^{2}}{M(p)}\right)\left\{\frac{(R-1) M(p)}{M(p)+2\left|a_{n}\right|}\right. \\
& \left.-\ln \left(1+\frac{(R-1) M(p)}{M(p)+2\left|a_{n}\right|}\right)\right\},
\end{aligned}
$$


which is Theorem 1.2 due to Govil [5].

By taking $\mu=1$ in inequality (2.1), we obtain the following results.

Corollary 2.5. If $p \in \mathcal{P}_{n}$ and $p(z) \neq 0$ for $|z|<k, k \geq 1$, then for $R>r \geq 1$,

$$
\begin{aligned}
\left|\left\{p\left(R e^{i \theta}\right)\right\}^{s}\right| \leq & \frac{\left(R^{n s}-r^{n s}\right)}{1+k}\{M(p)\}^{s}-\frac{n}{1+k}\{M(p)\}^{s}\left(1-\frac{(1+k)\left|a_{n}\right|}{M(p)}\right) h(n) \\
& +\left|\left\{p\left(r e^{i \theta}\right)\right\}^{s}\right|,
\end{aligned}
$$

where

$$
\begin{aligned}
h(n) & =\left(\frac{R^{n}-r^{n}}{n}\right)+\sum_{k=1}^{n-1}\left(\frac{R^{n-k}-r^{n-k}}{n-k}\right)(-1)^{k}\left(\frac{(1+k)\left|a_{n}\right|}{M(p)}+1\right)\left(\frac{(1+k)\left|a_{n}\right|}{M(p)}\right)^{k-1} \\
& +(-1)^{n}\left(\frac{(1+k)\left|a_{n}\right|}{M(p)}+1\right)\left(\frac{(1+k)\left|a_{n}\right|}{M(p)}\right)^{n-1} \ln \left(\frac{R(M(p))+(1+k)\left|a_{n}\right|}{r(M(p))+(1+k)\left|a_{n}\right|}\right)
\end{aligned}
$$

for $n \geq 1$ and $h(0)=0$.

Remark 2.6. We also have some other application Theorem 2.1, by taking $s=0$, $k=1$ and $r=1$ respectively.

\section{Lemmas}

For the proof of theorem, we need the following lemmas. Our first lemma is a well-known generalization of Schwarz's lemma (see for example [9, p. 167]).

Lemma 3.1. If $f(z)$ is analytic inside and on the circle $|z|=1, f(0)=a$, where $|a|<f$, then

$$
|f(z)| \leq M(f)\left(\frac{M(f)|z|+|a|}{|a||z|+M(f)}\right) .
$$

Lemma 3.2. If $p(z)=\sum_{v=0}^{n} a_{v} z^{v}$ is a polynomial of degree $n$, then for $|z|=R \geq 1$,

$$
|p(z)| \leq\left(\frac{\left|a_{n}\right| R+M(p)}{M(p) R+\left|a_{n}\right|}\right) M(p) R^{n} .
$$

The proof follows easily on applying Lemma 3.1 to the function $T(z)=z^{n} p(1 / z)$ and noting that $M(T)=M(p)$ (for details see [12, Lemma 2]).

From Lemma 3.2, one immediately gets:

Lemma 3.3. If $p(z)=\sum_{v=0}^{n} a_{v} z^{v}$ is a polynomial of degree $n$, then for $|z|=R \geq 1$,

$$
|p(z)| \leq\left(1-\frac{\left(M(p)-\left|a_{n}\right|\right)(R-1)}{M(p) R+\left|a_{n}\right|}\right) M(p) R^{n} .
$$

The following result is due to Chan and Malik [4]. 
Lemma 3.4. If $p(z)=a_{0}+\sum_{v=\mu}^{n} a_{v} z^{v}$ is a polynomial of degree $n$, and $p(z) \neq 0$ for $|z|<k, k \geq 1$, then

$$
\max _{|z|=1}\left|p^{\prime}(z)\right| \leq \frac{n}{1+k^{\mu}} M(p) .
$$

Lemma 3.5. If $p(z)=\sum_{v=0}^{n} a_{v} z^{v}$ is a polynomial of degree $n$, and let $r \geq 1$, then

$$
\left(1-\frac{\left(x-\left|a_{n}\right|\right)(r-1)}{r x+n\left|a_{n}\right|}\right) x
$$

is an increasing function of $x$, for $x>0$.

The proof of above lemma is straight forward using derivative test, so we omit the detail proof.

Lemma 3.6. Let

$$
h(n)=\int_{r}^{R} \frac{(t-1)\left(t^{n-1}\right)}{t+a} d t \text { for } n \geq 1
$$

Then

$$
\begin{aligned}
h(n) & =\left(\frac{R^{n}-r^{n}}{n}\right)+\sum_{k=1}^{n-1}\left(\frac{R^{n-k}-r^{n-k}}{n-k}\right)(-1)^{k}(a+1) a^{k-1} \\
& +(-1)^{n}(a+1) a^{n-1} \ln \left(\frac{R+a}{r+a}\right) .
\end{aligned}
$$

Proof. We define the function $f(n)=\int_{r}^{R} \frac{t^{n}}{t+a} d t$ for $n \geq 0$. It is easy to see that

$$
h(n)=f(n)-f(n-1) \text { for } n \geq 1 .
$$

We can obtain

$$
\begin{aligned}
f(n)+a f(n-1) & =\int_{r}^{R} \frac{t^{n}+a t^{n-1}}{t+a} d t \\
& =\int_{r}^{R} \frac{t^{n-1}(t+a)}{t+a} d t=\frac{R^{n}-r^{n}}{n}=g(n), \quad \text { (say). }
\end{aligned}
$$

Then

$$
f(n)=g(n)-a f(n-1) .
$$

Solving the recurrence relation (3.6), we get

$$
f(n)=\sum_{k=0}^{n-1} g(n-k)(-1)^{k} a^{k}+(-1)^{n} a^{n} f(0)
$$

where

$$
f(0)=\int_{1}^{R} \frac{1}{r+a} d r=\ln \left(\frac{R+a}{r+a}\right)
$$


Now, Substituting the value of $f(0)$ in $(3.7)$, we get

$$
f(n)=\sum_{k=0}^{n-1} g(n-k)(-1)^{k} a^{k}+(-1)^{n} a^{n} \ln \left(\frac{R+a}{r+a}\right), n \geq 0 .
$$

Using $h(n)=f(n)-f(n-1)$ and value of $g(n)$, we have Lemma 3.6 for $n \geq 1$.

Lemma 3.7. Let

$$
h(n)=\int_{r}^{R} \frac{(t-1)\left(t^{n-1}\right)}{t+a} d t \text { for } n \geq 1 .
$$

Then $h(n)$ is a non-negative increasing function of $n$ for $n \geq 1$.

Proof. Let

$$
f(n)=\int_{r}^{R} \frac{r^{n}}{r+a} d r \text { for } n \geq 0 .
$$

It is easy to see that $h(n)=f(n)-f(n-1)$ for $n \geq 1$. For $n \geq 1$, $f(n)-f(n-1)=\int_{1}^{R} \frac{(r-1)\left(r^{n-1}\right)}{r+a} d r \geq \int_{1}^{R} \frac{(r-1)\left(r^{n-2}\right)}{r+a} d r=f(n-1)-f(n-2)$ as $r^{n-1} \geq r^{n-2}$ for $r \geq 1$. Therefore,

$$
h(n)=f(n)-f(n-1) \geq f(n-1)-f(n-2)=h(n-1) .
$$

Therefore, $h(n)$ is an increasing function of $n$ for $n \geq 1$.

Also, $h(n)=f(n)-f(n-1) \geq 0$ for $n \geq 0$ as

$$
\int_{r}^{R} \frac{(t-1)\left(t^{n-1}\right)}{t+a} d r \geq 0
$$

for $n \geq 1$ and $h(0)=0$. Therefore, $h(n) \geq 0$ and is an increasing function of $n$ for $n \geq 0$.

\section{Proof of the Theorem}

Proof of Theorem 2.1. For each $\theta, 0 \leq \theta<2 \pi$, we have

$$
\begin{gathered}
\left|\left\{p\left(R e^{i \theta}\right)\right\}^{s}-\left\{p\left(r e^{i \theta}\right)\right\}^{s}\right|=\left|\int_{r}^{R} \frac{d}{d t}\left\{p\left(t e^{i \theta}\right)\right\}^{s} d t\right| \leq \int_{r}^{R} s\left|\left\{p\left(t e^{i \theta}\right)\right\}^{s-1}\right|\left|p^{\prime}\left(t e^{i \theta}\right)\right| d t \\
\leq\{M(p)\}^{s-1} \int_{r}^{R} t^{n s-n} s\left|p^{\prime}\left(t e^{i \theta}\right)\right| d t \\
\left|\left\{p\left(R e^{i \theta}\right)\right\}^{s}-\left\{p\left(r e^{i \theta}\right)\right\}^{s}\right| \\
\leq\{M(p)\}^{s-1} \int_{r}^{R} s t^{n s-1}\left\{1-\frac{\left(M\left(p^{\prime}\right)-n\left|a_{n}\right|\right)(t-1)}{n\left|a_{n}\right|+t M\left(p^{\prime}\right)}\right\} M\left(p^{\prime}\right) d t
\end{gathered}
$$

by using Lemma 3.3 for the polynomial $p^{\prime}(z)$, which is of degree $n-1$. We can see, from Lemma 3.5, the integrand in (4.1) is an increasing function of $M\left(p^{\prime}\right)$. 
Now, applying Lemma 3.4 to inequality (4.1), we get for $0 \leq \theta<2 \pi$,

$$
\begin{aligned}
& \left|\left\{p\left(R e^{i \theta}\right)\right\}^{s}-\left\{p\left(r e^{i \theta}\right)\right\}^{s}\right| \\
& \quad \leq\{M(p)\}^{s-1} \int_{r}^{R} s t^{s n-1}\left\{1-\frac{\left(\frac{n}{1+k^{\mu}} M(p)-n\left|a_{n}\right|\right)(t-1)}{n\left|a_{n}\right|+t \frac{n}{1+k^{\mu}} M(p)}\right\} \frac{n}{1+k^{\mu}} M(p) d t \\
& =\frac{\left(R^{n s}-r^{n s}\right)}{1+k^{\mu}}\{M(p)\}^{s}-\frac{n}{1+k^{\mu}}\{M(p)\}^{s}(1-a) \int_{r}^{R} \frac{(t-1)\left(t^{n-1}\right)}{t+a} d t,
\end{aligned}
$$

by taking $a=\frac{\left(1+k^{\mu}\right)\left|a_{n}\right|}{M(p)}$.

Using Lemma 3.6 in inequality (4.2), and substituting the value of $a$, we get

$$
\begin{aligned}
\left|\left\{p\left(R e^{i \theta}\right)\right\}^{s}\right| \leq & \frac{\left(R^{n s}-r^{n s}\right)}{1+k^{\mu}}\{M(p)\}^{s}-\frac{n}{1+k^{\mu}}\{M(p)\}^{s}\left(1-\frac{\left(1+k^{\mu}\right)\left|a_{n}\right|}{M(p)}\right) h(n) \\
& +\left|\left\{p\left(r e^{i \theta}\right)\right\}^{s}\right|,
\end{aligned}
$$

where

$$
\begin{aligned}
h(n) & =\left(\frac{R^{n}-r^{n}}{n}\right)+\sum_{k=1}^{n-1}\left(\frac{R^{n-k}-r^{n-k}}{n-k}\right)(-1)^{k}\left(\frac{\left(1+k^{\mu}\right)\left|a_{n}\right|}{M(p)}+1\right)\left(\frac{\left(1+k^{\mu}\right)\left|a_{n}\right|}{M(p)}\right)^{k-1} \\
& +(-1)^{n}\left(\frac{\left(1+k^{\mu}\right)\left|a_{n}\right|}{M(p)}+1\right)\left(\frac{\left(1+k^{\mu}\right)\left|a_{n}\right|}{M(p)}\right)^{n-1} \ln \left(\frac{R(M(p))+\left(1+k^{\mu}\right)\left|a_{n}\right|}{r(M(p))+\left(1+k^{\mu}\right)\left|a_{n}\right|}\right)
\end{aligned}
$$

for $n \geq 1$ and $h(0)=0$.

\section{Computation}

For the polynomial $p(z)=(z-2)^{2}, p(z) \neq 0$ for $|z|<1$ and $M(p)=9$. Then, for $R=3$, exact value of $M(p, R)$ is 25 . Using Theorem 1.2,

$$
M(p, R) \leq 45-7 *(2-11 / 9 \log (29 / 11))=39.29
$$

Using Corollary 2.2 of Theorem 2.1,

$$
M(p, R) \leq 45-7 *(4-22 / 9+22 / 81 \log (29 / 11))=32.26
$$

\section{References}

[1] Ankeny, N.C., Rivlin, T.J., On a theorem of S. Bernstein, Pacific J. Math, 5(2)(1955), 849-862.

[2] Aziz, A., Growth of polynomials whose zeros are within or outside a circle, Bull. Austral. Math. Soc., 81(1987), 247-256.

[3] Aziz, A., Mohammad, Q.G., Growth of polynomials with zeros outside a circle, Proc. Amer. Math. Soc., 81(1981), 549-553.

[4] Chan, T.N., Malik, M.A., On Erdös-Lax Theorem, Proc. Indian Acad. Sci., 92(1983), 191-193.

[5] Govil, N.K., On the maximum modulus of polynomials not vanishing inside the unit circle, Approx. Theory and its Appl., 5(3)(1989), 79-82. 
[6] Govil, N.K., On growth of polynomials, J. of Inequal. Appl., 7(5)(2002), 623-631.

[7] Govil, N.K., Qazi, M.A., Rahman, Q.I., Inequalities describing the growth of polynomials not vanishing in a disk of prescribed radius, Math. Inequal. Appl., 6(3)(2003), 491-498.

[8] Milovanović, G.V., Mitrinović, D.S., Rassias, Th.M., Topics in Polynomials: Extremal Problems, Inequalities, Zeros, World Scientific Publishing Co. Pte. Ltd., 1994.

[9] Nehari, Z., Conformal Mapping, McGraw-Hill, New York, 1952.

[10] Pólya, G., Szegö, G., Problems and Theorems in Analysis, Volume I, Springer-Verlag, Berlin-Heidelberg, 1972.

[11] Pukhta, M.S., Extremal Problems for Polynomials and on Location of Zeros of Polynomials, Ph. D Thesis, Jamia Millia Islamia, New Delhi, 1995.

[12] Rahman, Q.I., Schmeisser, G., Les Inégalitiés de Markov et de Bernstein, Les Presses de l'Université de Montréal, Montréal, Canada, 1983.

[13] Tripathi, D., On Extemal Problems and Location of Zeros of Polynomials, Ph.D Thesis, Banasthali University, Rajasthan, 2016.

Dinesh Tripathi

Department of Mathematics,

Manav Rachna University,

Faridabad-121001, India

e-mail: dinesh@mru.edu.in, dineshtripathi786@gmail.com 